\title{
Deferential vulnerability and patient decision-making
}

\author{
CEllis, MBBS, MRCS, MD, FCFP \\ Department of Family Medicine, School of Nursing and Public Health, University of KwaZulu-Natal, Durban, South Africa
}

Corresponding author: C Ellis (cristobalellis@gmail.com)

\begin{abstract}
'Autonomy is a fantasy. Our lives are inherently dependent on others and subject to forces and circumstances well beyond our control.' (Atul Gawande, Being Mortal)
\end{abstract}

S Afr J Bioethics Law 2017;10(2):56-57. DOI:10.7196/SAJBL.2017.v10i2.609

Deferential vulnerability arises when individuals subordinate themselves or submit to an authority figure when making decisions about their day-to-day lives and existence within their specific environment and relationships. This customary obedience occurs in cultures where certain hierarchical systems exist within the family or community. Vulnerable individuals may include, but are not limited to, women, children, the elderly and sometimes young adults.

Deferential vulnerability may occur within a medical relationship when patients defer to the authority of other family members or agencies when making health-related decisions. These decisions may not necessarily be involuntary or even be given unwillingly (that is, they may not necessarily be under direct duress or coercion), but the patient either makes decisions based on advice or directives from authority figures, or defers the decision in its entirety to such figures recognised by his or her cultural, community or religious contexts and customs. This can result in grey areas between acquiescence and voluntariness in the consent process. They often place the medical practitioner in a difficult situation as, on the one hand, the law requires consent from an individual patient or their legal proxy, and on the other hand, obtaining consent in an ethically sound manner requires consent to be a process in which effective decision-making is facilitated.

There is one further step that many African and indigenous communities worldwide may take in deferment, and that is to consult the ancestors or forefathers. Every now and then, the relatives of a patient may ask to take the patient home so that sacrifices (usually a goat or a cow) may be made to the ancestors, and also defer to a traditional healer to seek guidance in their consent deliberations.

Another form of deferential vulnerability may occur in more conservative traditional families. Female patients from these families are usually accompanied by a male member of the family, such as the husband, brother or father. Decisions are often made by the family as a community, as opposed to a Western individualistic decision that is left to the patient alone. In these particular circumstances, women may be vulnerable, but alternatively, may also be protected, depending on the specific dynamics within the family.

A recent case involved a 2-year-old Zulu child who needed a shunt owing to increasing intracranial pressure from a hydrocephalus. The mother was willing to give consent for the operation, but the father was unwilling, and the mother's mother (the child's grandmother) also objected to the operation. In South African law, only one parent needs to give consent for a child's operation. If there is a dispute, the key criterion is, what is in the best interests of the child? Zulu society is still, to some extent, patriarchal in nature, and the grandmother of a family also holds considerable authority concerning decisions over health matters. In this particular case, the mother, although willing, would not sign the consent forms out of deference to the authority of the father of her child and also the grandmother. This scenario demonstrates the potential impact of deferential vulnerability, as here the child was denied an important procedure because of concerns expressed by authority figures.

The influence of the family or community on individual choices is a subtle aspect of decision-making. This may not be as straightforward as it seems, because if I live in a society in which my partner makes all the decisions, and I am in agreement with this arrangement, then according to some ethicists, it is considered autonomous behaviour to let my partner make decisions on my behalf. We know that there are many subconscious influences at play when one consents to a procedure or operation, yet when people in groups are asked to make decisions they may come to very different conclusions than when asked to make such decisions individually. Although we all value information concerning our illnesses, we may not be as enthusiastic about making decisions for ourselves as theorists assume. In some situations we may prefer to let someone else decide for us. This is similar to the Sartrian view that man is condemned to be free, but does not necessarily enjoy the responsibility or burden of this freedom.

In obtaining informed consent one of the first steps is to check that the technological information has been relayed and understood by the patients and those assisting them. Even understanding the basic concepts and the words used to describe them is often difficult. The impasse with the child with rising intracranial pressure was solved by involving a traditional healer, along with the hospital counsellor, to explain the situation to the father and grandmother in a way and in a language that they could fully understand. In this way, cumbersome as it may seem, the authority figures in the child's and mother's life were brought into the decision-making process, thereby ensuring that the child's best interests were served.

We all surrender part of our decision-making rights in systems with which we are unfamiliar, or to persons with the relevant knowledge 
or power, be they lawyers, accountants or electricians. In addition, however well-informed we are, we often have problems subjectively balancing the risks and benefits of any action, especially when it comes to medical treatment.

While the ideal would be fully autonomous, uncoerced decisions, no one is fully autonomous - neither doctors nor patients. Despite these several restrictions and influences, we aspire to obtain the best quality of consent and decision-making for our patients. The question arises as to whether one uses Western ethical and communication models, or creates new models which are co-constituted by agreements between Western technological cultures and indigenous or traditional cultures. The best way forward may be to gradually introduce integrative models by increasing our communication skills and patient education, and also beyond the patient, to those in authority in their lives. This is not entirely possible without simultaneous social and educational change in the index populations.

Accepted 17 October 2017 